\title{
DEVELOPMENT OF A CONTINUOUS CHAMBER HIGH-GRADIENT MAGNETIC SEPARATOR WITH A STRONG FIELD
}

\author{
V.I. KARMAZIN ${ }^{\mathrm{a}}$, V.V. KARMAZIN ${ }^{\mathrm{b}, *}$, V.A. BARDOVSKIY ${ }^{\mathrm{b}}$ \\ and O.V. ZAMYTSKIY ${ }^{\mathrm{C}}$ \\ ${ }^{\mathrm{a}}$ The Mining Academy of the Ukraine, 19 Karl Marx Pr., 3200027 \\ Dnepropetrovsk, The Ukraine; ${ }^{b}$ Moscow State Mining University, \\ 6 Leninskiy Pr., 117935 Moscow, Russia; ${ }^{\mathrm{c}}$ Krivoy Rog Technical University, \\ 11 XX Partsiezda St., 50027 Krivoy Rog, The Ukraine
}

(Received 1 June 2000; accepted 23 July 2001; In final form 1 August 2001)

\begin{abstract}
High-gradient magnetic separation in a strong field is essentially a periodic process. In canister constructions it consists of separate operations, introduced by the electronic processor, while in rotary constructions the process is continuous. The separators with high capacity are very bulky, heavy and have massive rotors. All this reduces their technical and economic viability. The continuous process of high-gradient of separation in a chambered construction is reviewed. "Magnetic slipping" of feebly magnetic particles on prismatic elements of a matrix in the discharge zone utilized for the first time.
\end{abstract}

Keywords: High-gradient magnetic separation; Hydromechanical force; Hydrodynamic pressure; Interpolar space

\section{INTRODUCTION}

The process of high-gradient magnetic separation in a strong magnetic field was developed in 1935 in the USA. Institute "Mechanobr"

\footnotetext{
*Corresponding author.
} 
developed in 1940 a pilot-scale separator [1]. However, at that time, it failed to find industrial applications, apparently because its technological possibilities were underestimated and since clear requirements of industry in the field of magnetic beneficiation of fine-crushed materials were absent.

The process was revised 20 years later, after the patents of Jones and Carpenter [2,3] were filed. In the USSR at that time the problem of beneficiation of oxidized ferriferous quartzites, fine manganese and chrome ores, and of ores of rare metals gained importance. Numerous designs had appeared, and the separators underwent testing and were accepted for serial production by the State Commission of the Ministry of Ferrous Metallurgy of the USSR at the IV Session of the Central Mining-Dressing Industrial Complex. At the same time several types of seperators were bought such as DR-300 (Jones) for the Mikhailovskiy Mining and Dressing Industrial Complex, Boxmag-Rapid (England) separators for the Far East Mining and Chemical Industrial Complex, and others.

More than fifty powerful high-gradient separators, type 6ERM-3/ 300 (capacity up to $200 \mathrm{t} / \mathrm{h}$ ) were manufactured in the USSR (by "Gypromashugleobogaschenie") for the Dolinsk Mining and Dressing Industrial Complex of oxidized iron ores (the Ukraine), though the Complex was not commissioned because of the break-up of the USSR.

The above abundance of designs was accompanied by detailed theoretical, experimental and design investigations. At present the process of high-gradient magnetic separation is studied in detail, models are well established and the process is open to engineering calculations [4].

At the same time disadvantages of this technique and its drawbacks have become known, in particular:

- the dependence of the efficiency on the presence of strongly magnetic material in the pulp;

- low quality of the magnetic fraction as a result of the high concentration of fine weakly magnetic particles;

- comparatively high manufacturing cost;

- low recovery of weakly magnetic particles smaller than $10 \mu \mathrm{m}$, which is important, for instance in purification of kaolin. 
In "Mekhanobr" this process was called "magnet filtration" and this is what essentially it is. It is characterized by the dependence of the efficiency on the velocity of "filtration" of the pulp through the highgradient operating zone. That was convincingly shown by Lower [5] and by other investigators. Essentially the basis of this process is high-gradient magnetic settling surface (layers of balls, grooved plates, rods, and other media).

Comparative tests of matrices of various types were carried out by Rozhkov [6] who defined the field of their application. Investigations of the new matrices were also carried out by one of the authors [7].

According to these investigations, the matrices may be of two types. A contact type (e.g. balls, steel wool, etc.) allows to obtain very high recovery of weakly magnetic particles. However, these matrices require special regeneration. The other type is self-regenerating contactless matrices. These matrices can be divided into two types:

- Type I are matrices in which the operating elements (for example, grooved plates) are set across to the pulp flow.

- Type II are matrices in which the operating elements are set parallel to the pulp flow.

The type I matrices are more deeply studied and more widely used and applied, for example, in the well-known types of high-gradient magnetic separators such as DR-300, 6ERM-35/300 and others. In these matrices, the operating gaps rarely exceed $3 \mathrm{~mm}$, even under the highest values of the magnetic field. This results in frequent clogging of the matrix. In order to overcome this principal disadvantage it is possible to use type II, matrices which are used in this work.

One of the first designs of type II matrices are grates made of magnetic stainless steel, installed in a rotor along the magnetic flux (in the radial direction) and inclined by $28^{\circ}$ to the vertical. This design was used by Rapid Co. in their HW separators. This design practically excludes any possibility of the clogging of the matrices by large grains and allows a considerably greater yield of magnetic fraction per matrix mass.

We have continued our research in the direction of examining the technological possibilities of type II matrices, as the most stable during operation (absence of mechanical clogging) and allowing large loading of the magnetic product per unit mass of the matrix. 
Extraction of fine particles of weakly magnetic oxides and hydroxides of iron, manganese, chrome, nickel, also ferrites of tungstentitanium and others requires magnetic forces exceeding high values of dissipative forces of the medium resistance, particularly significant during the motion of fine particles. Hydrodynamic drag cannot be reduced by reducing the pulp velocity because of the production requirement of maximum throughput of a separator.

In most types of the above-mentioned ores regretfully fine comminution is inevitable in order to liberate the minerals, and if one takes into account the fact that magnetic properties of these minerals are of the same level, paramagnetic minerals, i.e. they are very small, then the increase in the magnetic force can be achieved only by increasing the magnetic field or its gradient. Hematite is essentially an antiferromagnetic and there are physical methods to increase magnetic properties as a result of magnetic and chemical interaction. This is beyond the scope of this work.

\section{THEORETICAL ANALYSIS OF POSSIBILITIES OF HIGH-GRADIENT MAGNETIC SEPARATION}

The use of traditional magnetic systems limits the level of intensity of the magnetic field and correspondingly the level of saturation of magnetisation of the core material of the magnetic system to $2 \mathrm{~T}$. Ferromagnetic matrices made of soft magnetic material, which as a result of high magnetic permeability of the material, are able to generate high gradients of the magnetic field offer many more possibilities.

Such an approach is applicable when using as a source of the external field either conventional electromagnetic system or superconducting magnets. However, one should bear in mind that at strong magnetic fields approaching $2 \mathrm{~T}$, a further increase of intensity will cause magnetic saturation of the ferromagnetic matrices and will reduce the effect of their shape on the ponderomotive force in the gap. Such a situation requires small matrix elements comparable to the size of separated particles, for instance, steel wool. These polar elements (wires, meshes, etc.) even if they are completely magnetically saturated retain a high level of local gradients as a result of superposition of 
contributions of its magnetic induction increasing thus the field gradient and the ponderomotive magnetic forces near its surface.

$$
B_{M}=H_{e}+\left.J\right|_{\left(B \rightarrow B_{s}\right)}=H_{e}+J_{s},
$$

so that $J_{s}=$ const.

$$
\operatorname{grad} H_{e}=\left(H_{e}+J_{s}-H_{e}\right) / \Delta x=J_{s} / \Delta x
$$

where

- $B_{m}$ is the magnetic induction of the matrix;

- $H_{e}$ is the intensity of the external magnetic field;

- $B_{s}$ and $J_{s}$ are the magnetic induction and magnetisation of the matrix material and

$-\Delta x$ is the height of the contact capture zone of a matrix element.

It follows from the Eq. (2) that when $J=J_{s}$ the field gradient has the maximum value. However, when $H_{e}$ increases further the field gradient will decrease since $J_{s}=$ const.

These conclusion have a great significance for the high-gradient magnetic separation, as the magnetic saturation of mineral particles as a rule, starts at lower values of $H_{e}$, afterwards the contribution of magnetic properties of the particles to the value of the magnetic force does not increase further. It can be easily seen from the classical formula for the ponderomotive (traction) magnet force [7]:

$$
F_{\text {magn }}=M_{p} \operatorname{grad} H_{e}=J_{p} V_{p} \operatorname{grad} H_{e}=V_{p} \kappa_{p} H_{i} \operatorname{grad} H_{e}=\chi_{p} m H_{i} \operatorname{grad} H_{e},
$$

where

$\begin{array}{ll}M_{p} & \text { is the magnetic moment of a particle, } \\ J_{p} & \text { is the magnetisation of a particle, } \\ V_{p} & \text { is the volume of a particle, } \\ \kappa_{\mathrm{p}}=J_{p} / H_{i} & \text { is the volumetric magnetic susceptibility, } \\ \chi_{p}=\kappa_{p} / \delta & \text { is the specific magnetic susceptibility, } \\ \delta & \text { is the density of a mineral particle, } \\ H_{i} & \text { is the intensity of the internal magnetic field of a particle, } \\ H_{e} & \text { is the intensity of the external magnetic field of a particle, } \\ m & \text { is the mass of a particle. }\end{array}$


Taking into account that when $B_{e} \rightarrow B_{s}$ then $J_{p} \rightarrow J_{s}$. In other words under magnetic saturation of a mineral particle in a strong external field $H_{e}$ the magnetisation of a particle $J_{p}=\left.\kappa_{p} H_{i}\right|_{\left(J \rightarrow J_{s}\right)}$ becomes constant. However, when $H_{e}$ increases, $\kappa_{p}$ can decrease, and $H_{i}$ will somewhat increase, since $H_{i}=$ const. Overall, however, after the magnetic saturation is achieved, the contribution of intensity of the internal field of the particle $H_{i}$ does not increase any more and a further increase of the magnitude of the magnetic force can be achieved only by increasing grad $H_{e}$, irrespective whether $H_{e}$ increases or not. The particle size of refractory ores decreases with time, and therefore the need to increase the forces in the separators is evident [8].

The first design of the Jones separators were of the chamber type and featured several technological advantages:

- a high, controlled pressure of the feed and of the wash water at the entry into the chamber;

- a minimum quantity of high-gradient matrices;

- compactness and the absence of a complicated and powerful drive, etc.

At the same time cyclic separators have their own inherent shortcomings associated with the complexity of control of the multiphase cycle, low efficiency and others. In the present work the aim was to combine advantages of the two designs and to avoid the known shortcomings as much as possible.

The process of separation in the proposed design is performed in the following way.

A weakly magnetic pulp is supplied to the entrance of the operating chamber at a given pressure, along the settling surfaces of ferromagnetic bodies. In the chamber, the magnetic field is being generated with decreasing intensity in the direction of the pulp movement. When the pulp enters the magnetic field, separation of particles takes place. Weakly magnetic particles under the action of ponderomotive forces settle on the working surfaces of the ferromagnetic bodies, and non-magnetic particles under the action of gravity and hydrodynamic forces sink. Non-magnetic particles thus pass through the gaps between ferromagnetic bodies, and are thus removed from the working space of the separator. It is assumed that the force that depends on the field 
gradient in the working gap achieves the value of the forces between the particles and polar elements of the matrix. The weakly magnetic particles that settled in the capture zone of the ferromagnetic bodies are under the action the magnetic cohesive forces and of the hydrodynamic forces of the pulp flow. The resistance of particles against shear stress from the matrix exceeds by a factor of 10 to 2 their sliding resistance along the matrix.

The value of the hydrodynamic force of the pulp pressure $F_{h . f}$ along the settling surfaces of ferromagnetic bodies when the pulp enters the chamber is given by:

$$
F_{h . f .}=p S F_{r s}=k\left(B^{2} s f\right) / 2 \mu_{o},
$$

where:

$p \quad$ is the pressure in the initial part of chamber, $(\mathrm{Pa})$

$S \quad$ is the cross section area of the chamber, $\left(\mathrm{m}^{2}\right)$

$F_{r s}$ is the resistance force to the magnetic sliding of particles over the working surfaces of ferromagnetic bodies, (N)

$k \quad$ is the adhesion coefficient of particles to the ferromagnetic bodies,

$B \quad$ is the value of the magnetic induction, (T)

$S \quad$ is the area of the contact surface of particles with a body, $\left(\mathrm{mm}^{2}\right)$

$\mu_{o}$ magnetic permeability of vacuum,

$f \quad$ is the coefficient of friction of sliding of weakly magnetic particles over the operating surfaces of ferromagnetic bodies.

Weakly magnetic particles, after their settling onto the ferromagnetic bodies under the action of force $F_{r . m}$, move towards the discharge by magnetic sliding. When the magnetic particles are moving over the settling surface from the chamber entrance to its exit, the hydrodynamic force of the pulp flow decreases because of the removal of the pulp flow containing non-magnetic particles through the gaps between the ferromagnetic bodies out of the working space of the chamber. To ensure continuous displacement of the magnetic particles over ferromagnetic bodies the field of the magnetic system between the ferromagnetic bodies of the separator is set with different intensity, decreasing in the direction of the pulp movement. The field intensity between the ferromagnetic bodies along the entire length of the 
chamber is chosen is such a way that at every moment of the pulp displacement the condition (4) is observed.

Outside the interpolar space of the magnetic system the intensity of the magnetic field approaches zero. The cohesive forces of magnetic particles with the ferromagnetic bodies in this part of the chamber also approach zero. Then, under the gravitational and hydrodynamic forces the magnetic fraction is being removed from the ferromagnetic bodies and is continuously collected in a separate container.

\section{SPECIAL FEATURES OF THE DESIGN OF THE SEPARATOR}

The high-gradient chamber continuous magnetic separator consists of a magnetic system 1 in the interpolar space of which an operating chamber 2 is installed. This chamber contains an assembly of parallel ferromagnetic prisms 3 . Ribs and edges of the neighboring prisms form pairs of poles in the direction of the magnetic flux. The pairs of the poles are evenly distributed along the cross section of the chamber as shown in Fig. 1.

The cross section of the prisms decreases in the direction of pulp movement, and their end parts are fixed outside interpolar space of the magnetic systems. The uniformity of distribution of the prisms is guaranteed by constancy of the step between their centers of mass along the entire length of the chamber.

Prisms of small cross-section are fixed to the walls of the chamber to avoid their mutual attraction, in the magnet field. The number of the prism edges may be different, for instance 3 or 4 , as shown in Fig. 2. The upper edge of every prism coincides with the direction of the magnetic flux, which is required for uniform distribution of the pulp over the entire length of chamber. The upper part of the chamber houses the feed pipe 4 . The feed rate into working space of the chamber is controlled by a gate 5 , located at the entrance into the chamber above the upper row of the prisms. In the bottom part of the chamber a control limiting slide valve 6 is installed. The bottom 7 of the chamber under the interpolar space of the magnetic system is inclined in the direction of the pulp movement. Containers of the 


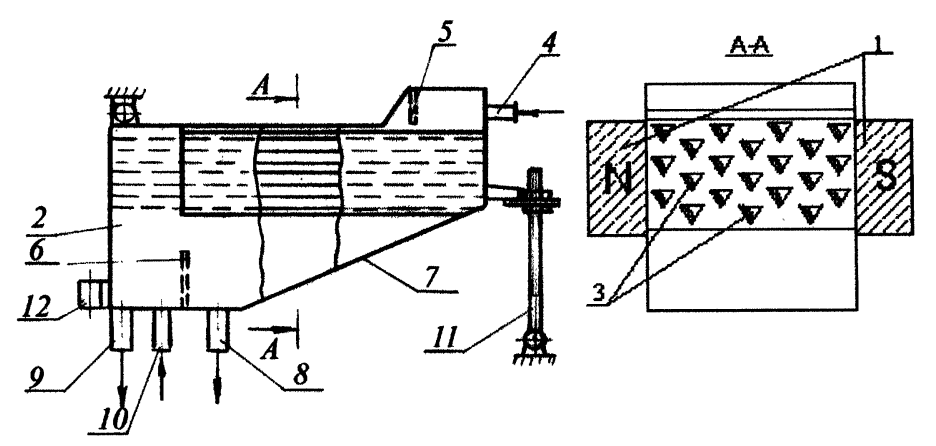

FIGURE 1 A schematic diagram of the high-gradient high intensity chamber continuous magnetic separator.
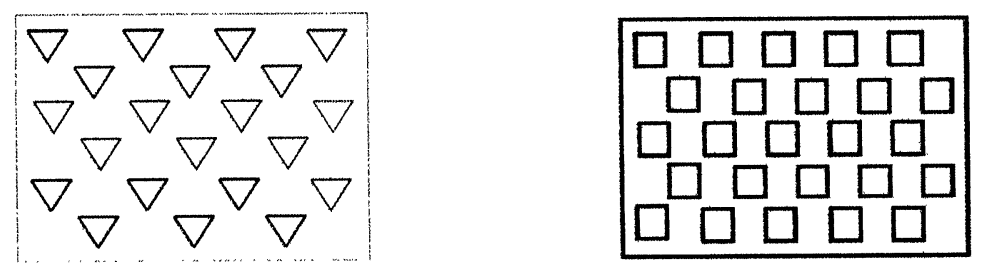

FIGURE 2 The cross section of the matrices, used in this experiment.

non-magnetic fraction 8 and the magnetic fraction 9 are located on the horizontal part as the bottom. Between the container of the magnetic fraction and the slide valve a connecting pipe 10 of the water supply is installed. It is used to provide additional cleaning of prism ends from the entrained magnetic particles. The chamber is installed in such a way that it is possible to control its inclination angle in the vertical plane, for instance by means of a screw device 11 .

The device is also provided with a vibrator 12 , fastened on the end part of the chamber body.

The separator operates in the following way. In the working interpolar space of the chamber a weakly magnetic pulp is delivered through the feed pipe 4 under a set pressure. Its quantity is regulated by gate 5 and the pulp reaches the settling surface of prisms 3, as shown in Fig. 3.

Under the action of the ponderomotive forces a portion of magnetic particles settles onto the working surfaces of the upper row of the 
The pulp motion direction

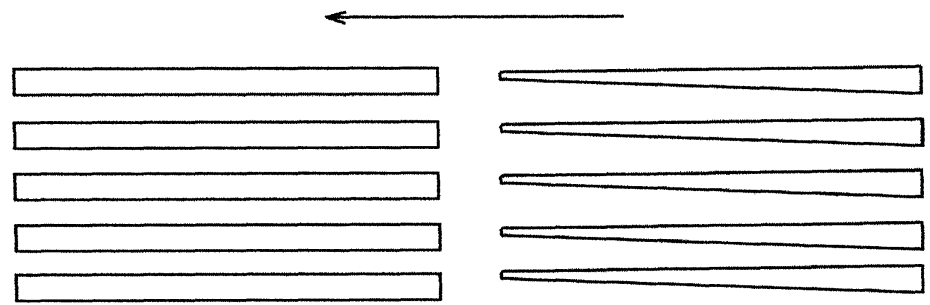

FIGURE 3 The top view of the matrices: (a) constant section; (b) decreasing section.

prisms. The remaining particles, together with non-magnetic particles in the pulp, pass through the gaps between the upper row of the prisms. Under the ponderomotive forces these particles reach the working surfaces of the bottom row of the prisms, where the setting of the magnetic particles is taking place in the entire volume of the chamber. The non-magnetic particles under the gravitational and hydrodynamic forces pass through the gaps between the prisms and along the inclined bottom they enter the container of the non-magnetic fraction.

The high field gradient in gaps between the poles formed by ribs and edges of the adjacent prisms ensures a sufficiently complete settling of weakly magnetic particles on the working surfaces. The settled magnetic particles under the force of the hydrodynamic pressure of the pulp move along the prisms towards the end of the chamber, and leave the interpolar space of the magnetic system. Under the gravitational and hydrodynamic forces, after being torn away from the prisms they enter the container of the magnetic fraction. The increase of the gaps between the prisms during the sliding of the magnetic particles facilitates their removal from the end parts of the prisms and their cleaning from the non-magnetic particles.

The efficiency of the magnetic separation is increased by controlling the inclination angle of the chamber using the screw device 11 .

The installation of a vibrator reduces the attractive forces of the magnetic particles to the end parts of the prisms and, improves thus their cleaning from the magnetic particles. The parameters of vibration are chosen in such away that the removal of the magnetic particles from the prisms takes place beyond the limits of interpolar space of the magnetic system. 


\section{THE EXPERIMENTAL INVESTIGATIONS}

The magnetic system of the laboratory isodynamic separator SIM-1 was used for experiments. The magnetic system with the help of two pilot wheels through special reducers can be set at any angular position, which is of great importance when choosing an optimal regime of separation. The pulp was supplied to the separator under pressure by a centrifugal pump and both the magnetic and the nonmagnetic products were returned to the pump hopper. This provided a continuous regime of the rig for relatively small samples of about one kilogram. The initial concentration of solids in the pulp was, as is usual in wet separation, between 20 and $25 \%$. During separation the concentration decreased somewhat as a result of the "support" water supplied to control the process. The main controlled parameters that affect the efficiency of the separator BKHMC are the following:

1. the magnetic induction in the working space of the separator,

2. the pressure in the feed pipe of the separator,

3. the concentration of solids in the pulp,

4. the inclination angle of the chamber (between $+12^{\circ}$ and $-12^{\circ}$ ) with respect to the horizontal plane,

5. size of the nozzles on the discharge pipes for the non-magnetic and magnetic products.

All the other parameters that were not controlled (type and design of the matrix pattern of the magnetic field, different chambers, etc.) were set when the chamber was manufactured and they are not controlled in the concrete design.

When planning experiments to determine the optimal parameters, the five above-specified parameters were take into account. The separator chamber, in which the experimental investigations were carried out corresponds to Fig. 1, and its dimensions: height, width, and the length are equal to 50,40 and $200 \mathrm{~mm}$, respectively. Bars, used as the matrix have a cross section of an equilateral triangle with $3 \mathrm{~mm}$ side, made of "Armco" iron.

The magnetic minerals used in the initial sample were weakly magnetic oxides of manganese such as pyrolusite, and psilomelane, having a rather well defined magnetic susceptibility of $1 \cdot 10^{-6} \mathrm{~cm}^{3} / \mathrm{g}$, in contrast to weakly magnetic iron oxides. The sample was taken 
TABLE I The results of separation of the manganese oxides

\begin{tabular}{lccc}
\hline Product & $M n(\%)$ & Yield $(\%)$ & Recovery $(\%)$ \\
\hline Magnetic product & 41.5 & 44.3 & 87.5 \\
Non-magnetic product & 4.7 & 55.7 & 12.5 \\
Feed & 21.0 & 100.0 & 100.0 \\
\hline
\end{tabular}

from the Porozhnenski deposit (Russia), was crushed to less than $0.1 \mathrm{~mm}$, and then it was pre-separated on a standard laboratory magnetic separator $138 \mathrm{CE}$ with magnetic induction of $0.5 \mathrm{~T}$ in order to remove strongly and medium magnetic minerals. The results of separation using the separator BKHMC obtained using the optimal parameters $\left(\mathrm{B}=1.2 \mathrm{~T}, \alpha=+8^{\circ}\right)$, are shown in Table $\mathrm{I}$.

\section{CONCLUSIONS}

In the Laboratory of magnetic and electrical methods of the scientific research centre of the Moscow State Mining University a new process has been developed. In this process the magnetic fraction "slips" under the pulp pressure into a discharging zone. Thus, the creation of a highgradient continuous chamber magnetic separator has been a success.

As far as its technological efficiency is concerned the new separator exceeds considerably to the existing HGMS units in issues of the efficiency per unit mass of separator and consumption of electric energy per ton of pulp.

A complicated manual control of the process is a disadvantage of this separator and a system of automatic control is being developed in the laboratory.

\section{References}

[1] V.G. Derkach and I.S. Datshuk (1947). Electromagnetic processes of enrichment, Metallurgizdat.

[2] Patent specification GB No. 768,451,B 03 C, 1954.

[3] Patent specification USA No. 3,375,925, B 03 C, 1968.

[4] J. Svoboda (2001). Favoured theoretical models of high-gradient magnetic separation: fallacy or reality? Miner. Eng., (to be published).

[5] I.E. Louwer, T.L. Wright and H.R. Kokal (1968). The behavior of Mesabi iron and silicate minerals in $20 \mathrm{kG}$ magnetic field. SME Transaction, 241, 194. 
[6] I.M. Rozhkov (1988). Increase of stability and efficiency of the processes of high gradient separation by means of using of settling surfaces with alongitudinal magnetic flow. Dissertation for Candidate of Technical Science Degree (PhD.), NC GMI, Ordzhonikidze, USSR.

[7] V.V. Karmazin (1998). Magnetic and electric methods of separation in solving ecological problems of mining production. In: Ecological Problems of Mining Production, Treatment and Distribution of Wastes. MI and RSA, Moscow, Russia.

[8] V.V. Karmazin (1997). Theoretical assessment of technological potential of magnetic and electrical separation. Magn. Electr. Sep., 8, 139.
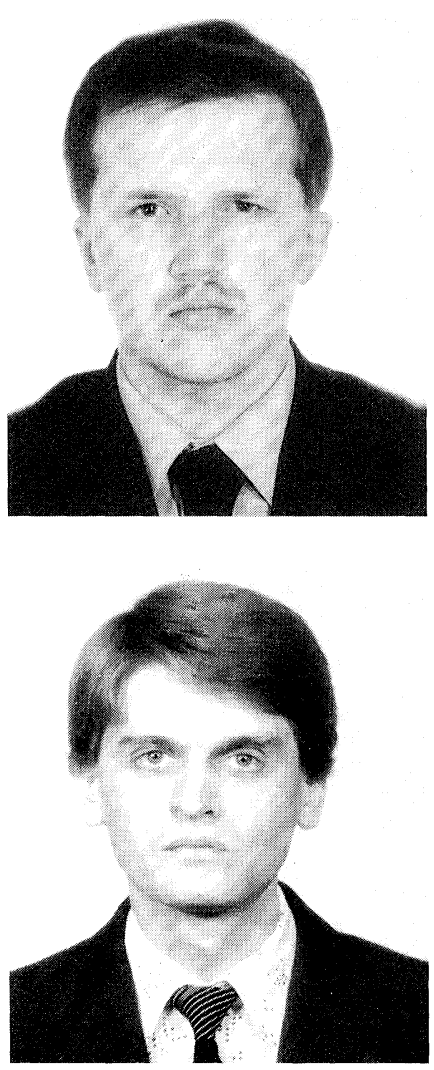

Vitaliy Ivanovich Karmazin: for biography see Magn. Electr. Sep., 3 (1992), 90.

Viktor Vitalyevich Karmazin: for biography see Magn. Electr. Sep., 8(1997), 159.

\section{Vladimir Anatolievich Bardovskiy was} born in 1973 and in 1995 he graduated as a mechanical engineer from the Department of Engineering Technology of the Moscow Mining Institute. At present he is a post-graduate student at the Moscow State Mining University. His main area of interest is in development of new products with special emphasis on high-gradient magnetic separation.

Oleg Vladimirovich Zamytskiy was born in 1962 and in 1984 he graduated from the Krivoy Rog Mining Institute. In 1995 he obtained his Ph.D. (candidate of sciences) degree from the same institution. In 1996 he was appointed the associate professor at the Department of the Heat and Power Engineering of the Krivoy Rog Technical University. 